\title{
STRESS DISTRIBUTION IN A COMPOSITE COATING BY LOCAL LOADING OF THE FREE SURFACE
}

\author{
Roman KULCHYTSKY-ZHYHAILO*, Waldemar KOŁODZIEJCZYK \\ "Faculty of Mechanical Engineering, Bialystok University of Technology \\ ul. Wiejska 45C, 15-351 Białystok, Poland \\ r.kulczycki@pb.edu.pl, w.kolodziejczyk@pb.edu.pl
}

\begin{abstract}
A three-dimensional problem of the theory of elasticity for halfspace with multilayered coating with periodical structure is considered. The fundamental layer consists of two layers with different thicknesses and different mechanical properties. The coating is described by the homogenized model with microlocal parameters. The solution is derived by using integral Fourier transform. Calculations were conducted with the assumption of elliptical distribution of normal and tangential tractions applied to the surface of the layered system in a circular area. Analysis of the stresses was restricted to the first principal stress distribution.
\end{abstract}

Key words: Gradient Coating, Three-Dimensional Problem of Elasticity, Normal Loading, Shear Loading

\section{INTRODUCTION}

In the mechanics of contact interaction, much attention is now given to coatings used for the improvement of the tribological characteristics of friction couples. Thus, the coatings formed by periodically deposited elastic layers are now extensively investigated (Farhat et al., 1997; Voevodin et al., 2001) parallel with the uniform coatings (Schwarzer 2000; Bargallini et al., 2003; Kulchytsky-Zhyhailo and Rogowski, 2010) and nonuniform coatings whose mechanical properties are described by continuous functions of the distance from the surface (Guler and Erdogan, 2007; Liu et al., 2008; Kulchytsky-Zhyhailo and Bajkowski, 2010). In the analysis of the stressed state, the researchers, as a rule, focus their attention on the evaluation of the tensile and HuberMises stresses described by the second invariant of the deviator of the stress tensor.

Considering contact problems for multilayered coatings in classical approach we need to solve partial differential equations for every sublayer and satisfy continuity conditions on the interfaces.

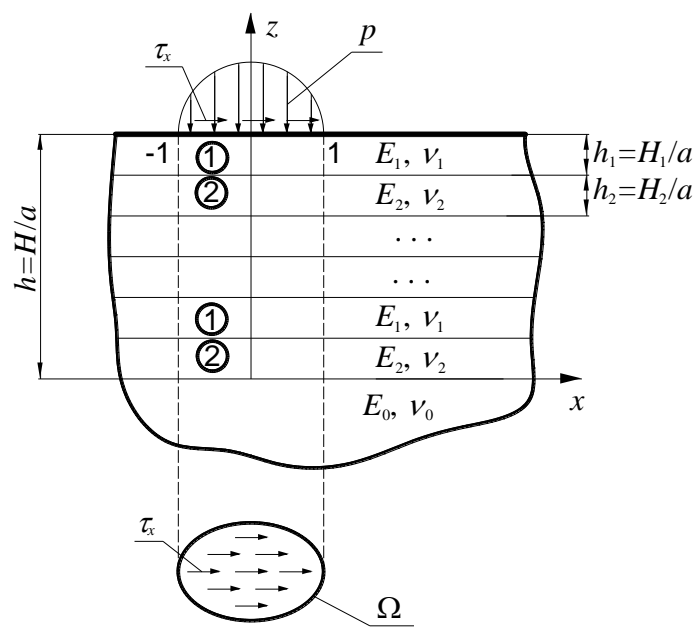

A coating with periodical structure can be replaced by a homogeneous one by using e.g. homogenized model with microlocal parameters (Matysiak and Woźniak, 1987; Woźniak, 1987).

As it was show in earlier papers (Kołodziejczyk and Kulchytsky-Zhyhailo, 2013; Kulchytsky-Zhyhailo and Kołodziejczyk, 2005; Kulchytsky-Zhyhailo, 2011) the stress distribution in the substitute homogeneous medium is a good approximation of stress distribution in the multilayered medium when the ratio of thickness of a fundamental layer to a specific size of contact area is less than 0.1 .

In the present work, we consider a three-dimensional problem of elastic half space with laminated coating of periodic structure loaded by normal and tangential tractions.

The multilayered coating is replaced by a homogeneous one which mechanical properties are described by the homogenized model with microlocal parameters. The objective of this work is to determine relations between applied loading and stresses in the coating and in the substrate as well as analysis of tensile stresses and Huber-Mises stresses in the coating due to loading applied in a circular area on the free surface.

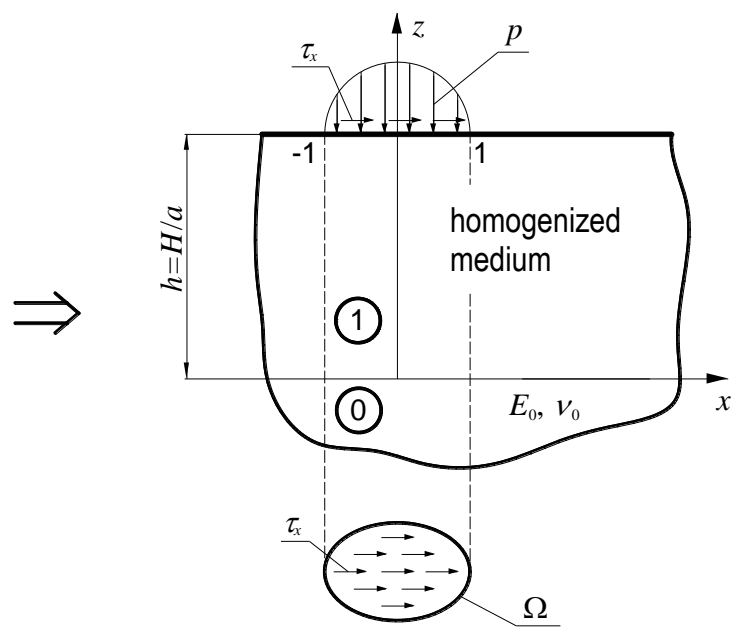

Fig. 1. The scheme of the problem 


\section{FORMULATION OF THE POBLEM}

Let us consider a non-uniform elastic half-space loaded by normal $p$ and tangential $\tau_{x}$ traction in the region $\Omega$ with the specific size $a$ on the free surface (Fig. 1). The non-uniform half space is formed by the homogeneous isotropic half space with Young's modulus $E_{0}$ and Poisson's ratio $v_{0}$ and a system of two periodically deposited elastic layers with thicknesses $H_{1}$ and $H_{2}$ ( $H=H_{1}+H_{2}$ is the thickness of the fundamental layer), Young's moduli $E_{1}$ and $E_{2}$, and Poisson's ratios $v_{1}$ and $v_{2}$, respectively. Assume that the conditions of perfect mechanical contact are realized between the layers of the coating and between the coating and the substrate.

Since the nonhomogeneous coating is described by the homogenized model with microlocal parameters (Matysiak and Woźniak, 1987; Woźniak, 1987) the governing equations take the form:

$$
\begin{aligned}
& A_{1} u_{x, x x}^{(1)}+ 0.5\left(A_{1}-A_{2}\right) u_{x, y y}^{(1)}+A_{5} u_{x, z z}^{(1)}+ \\
&+0.5\left(A_{1}+A_{2}\right) u_{y, x y}^{(1)}+\left(A_{3}+A_{5}\right) u_{z, x z}^{(1)}=0, \\
& 0.5\left(A_{1}-\right.\left.A_{2}\right) u_{y, x x}^{(1)}+A_{1} u_{y, y y}^{(1)}+A_{5} u_{y, z z}^{(1)}+ \\
&+0.5\left(A_{1}+A_{2}\right) u_{x, x y}^{(1)}+\left(A_{3}+A_{5}\right) u_{z, y z}^{(1)}=0, \\
& A_{5}\left(u_{z, x x}^{(1)}+u_{z, y y}^{(1)}\right)+A_{4} u_{z, z z}^{(1)}+\left(A_{3}+A_{5}\right)\left(u_{x, x z}^{(1)}+u_{y, y z}^{(1)}\right)=0,
\end{aligned}
$$

where $\mathbf{u}^{(1)}$ - macro-displacements vector (displacements averaged in a fundamental layer), $x, y, z$ - dimensionless coordinates (Cartesian coordinates related to a specific size of contact area a), $f_{, k}=\partial f / \partial k, k=x, y, z, A_{i}, i=1,2, \ldots, 5$ - coefficients calculated from known relations (Matysiak and Woźniak, 1987; Kaczyński, 1994; Kulchytsky-Zhyhailo, 2011; Kołodziejczyk and Kulchytsky-Zhyhailo, 2013) on the base of mechanical and geometrical properties of alternating layers:

$$
\begin{aligned}
& A_{1}=\tilde{\lambda}+2 \tilde{\mu}-\frac{[\lambda]^{2}}{\hat{\lambda}+2 \hat{\mu}}, A_{2}=\tilde{\lambda}-\frac{[\lambda]^{2}}{\hat{\lambda}+2 \hat{\mu}}, \\
& A_{3}=\tilde{\lambda}-\frac{[\lambda]([\lambda]+2[\mu])}{\hat{\lambda}+2 \hat{\mu}}, \\
& A_{4}=\tilde{\lambda}+2 \tilde{\mu}-\frac{([\lambda]+2[\mu])^{2}}{\hat{\lambda}+2 \hat{\mu}}, A_{5}=\tilde{\mu}-\frac{[\mu]^{2}}{\hat{\mu}}, \\
& \tilde{\lambda}=\eta \lambda_{1}+(1-\eta) \lambda_{2}, \tilde{\mu}=\eta \mu_{1}+(1-\eta) \mu_{2}, \\
& {[\lambda]=\eta\left(\lambda_{1}-\lambda_{2}\right),[\mu]=\eta\left(\mu_{1}-\mu_{2}\right),} \\
& \hat{\lambda}=\eta \lambda_{1}+\frac{\eta^{2}}{1-\eta} \lambda_{2}, \hat{\mu}=\eta \mu_{1}+\frac{\eta^{2}}{1-\eta} \mu_{2}, \eta=\frac{H_{1}}{H}, \\
& \lambda_{i}=\frac{E_{i} v_{i}}{\left(1+v_{i}\right)\left(1-2 v_{i}\right)}, \mu_{i}=\frac{E_{i}}{2\left(1+v_{i}\right)} .
\end{aligned}
$$

Equations (1) have the same form as equations of theory of elasticity for a transversely isotropic solid. Therefore their solution can be expressed in terms of elastic potentials proper to a transversely isotropic medium (Elliot, 1949):

$$
\begin{aligned}
& u_{x}^{(1)}=\Psi_{1, x}+\Psi_{2, x}+\Psi_{3, y}, u_{y}^{(1)}=\Psi_{1, y}+\Psi_{2, y}-\Psi_{3, y}, \\
& u_{z}^{(1)}=\kappa_{1} \Psi_{1, z}+\kappa_{2} \Psi_{2, z},
\end{aligned}
$$

where elastic potentials satisfy equations:

$$
\Psi_{i, x x}+\Psi_{i, y y}+\gamma_{i}^{2} \Psi_{i, z z}=0,
$$

$\gamma_{i}^{2}, i=1,2$ are roots of the characteristic equation:

$$
\begin{aligned}
& A_{1} A_{5} \gamma^{4}+\left(A_{3}^{2}+2 A_{3} A_{5}-A_{1} A_{4}\right) \gamma^{2}+A_{4} A_{5}=0, \\
& \kappa_{i}=\frac{\gamma_{i}^{2} A_{1}-A_{5}}{A_{3}+A_{5}}, \gamma_{3}^{2}=\frac{2 A_{5}}{A_{1}-A_{2}} .
\end{aligned}
$$

Considered boundary problem, formulated in dimensionless coordinates related to the specific size $a$ of the loading area, leads to equations of theory of elasticity (2b) defined in the appropriate homogenized medium and equations

$$
\left(1-2 v_{0}\right) \Delta \mathbf{u}^{(0)}+\operatorname{grad} \operatorname{div} \mathbf{u}^{(0)}=0
$$

defined in the substrate, with boundary conditions:

- normal and tangential tractions on the surface,

$\sigma_{z z}^{(1)}(x, y, z)=-p(x, y), \sigma_{x z}^{(1)}(x, y, h)=\tau_{x}(x, y)$,

$\sigma_{y z}^{(1)}(x, y, h)=0,(x, y) \in \Omega$,

- ideal contact between coating and substrate,

$u_{x}^{(0)}(x, y, 0)=u_{x}^{(1)}(x, y, 0), u_{y}^{(0)}(x, y, 0)=u_{y}^{(1)}(x, y, 0)$,

$u_{z}^{(0)}(x, y, 0)=u_{z}^{(1)}(x, y, 0)$,

$\sigma_{z z}^{(0)}(x, y, 0)=\sigma_{z z}^{(1)}(x, y, 0), \sigma_{x z}^{(0)}(x, y, 0)=\sigma_{x z}^{(1)}(x, y, 0)$,

$\sigma_{y z}^{(0)}(x, y, 0)=\sigma_{y z}^{(1)}(x, y, 0)$,

- vanishing displacement components:

$u_{x}^{(i)}, u_{y}^{(i)}, u_{z}^{(i)} \rightarrow 0, x^{2}+y^{2}+z^{2} \rightarrow-\infty, i=0,1$,

where $\mathbf{u}^{(0)}$ is the displacement vector in the substrate.

The characteristic feature of the homogenized model is that it gives different expressions for calculation of the stress tensor components, which experience a jump on the interfaces between layers.

$$
\begin{aligned}
& \sigma_{x x}^{(1, k)}=K_{k} u_{x, x}^{(1)}+L_{k} u_{y, y}^{(1)}+M_{k} u_{z, z}^{(1)}, k=1,2, \\
& \sigma_{y y}^{(1, k)}=L_{k} u_{x, x}^{(1)}+K_{k} u_{y, y}^{(1)}+M_{k} u_{z, z}^{(1)}, k=1,2, \\
& \sigma_{x y}^{(1, k)}=\mu_{k}\left(u_{x, y}^{(1)}+u_{y, x}^{(1)}\right), k=1,2,
\end{aligned}
$$

where $\sigma^{(1, k)}$ is the stress tensor in $k$ - th layer of the fundamental layer $(k=1,2), K_{k}, L_{k}, M_{k}$ - coefficients calculated from known expressions (Kaczyński, 1994):

$$
\begin{aligned}
& K_{k}=\lambda_{k}+2 \mu_{k}-h_{k} \lambda_{k} \frac{[\lambda]}{\hat{\lambda}+2 \hat{\mu}}, L_{k}=\lambda_{k}-h_{k} \lambda_{k} \frac{[\lambda]}{\hat{\lambda}+2 \hat{\mu}}, \\
& M_{k}=\lambda_{k}-h_{k} \lambda_{k} \frac{[\lambda]+2[\mu]}{\hat{\lambda}+2 \hat{\mu}}, h_{1}=1, h_{2}=-\frac{\eta}{1-\eta} .
\end{aligned}
$$

It is essential to note that discussed stresses differ significantly in the individual sublayers composing a fundamental layer (Kulchytsky-Zhyhailo, 2011; Kulchytsky-Zhyhailo and Kołodziej- 
czyk, 2005; Kołodziejczyk and Kulchytsky-Zhyhailo, 2013).

The remaining stress tensor components can be obtained using the following relations:

$$
\begin{aligned}
& \sigma_{z z}^{(1)}=\sigma_{z z}^{(1, k)}=A_{3}\left(u_{x, x}^{(1)}+u_{y, y}^{(1)}\right)+A_{4} u_{z, z}^{(1)}, k=1,2, \\
& \sigma_{x z}^{(1)}=\sigma_{x z}^{(1, k)}=A_{5}\left(u_{x, z}^{(1)}+u_{z, x}^{(1)}\right), k=1,2, \\
& \sigma_{y z}^{(1)}=\sigma_{y z}^{(1, k)}=A_{5}\left(u_{y, z}^{(1)}+u_{z, y}^{(1)}\right), k=1,2 .
\end{aligned}
$$

\section{SOLUTION OF THE POBLEM}

General solution of differential equations defined in the coating and in the substrate we obtain using two-dimensional Fourier transform:

$\tilde{f}(\xi, \eta, z)=\frac{1}{2 \pi} \int_{-\infty}^{+\infty} \int f(x, y, z) \exp (-i x \xi-i y \eta) d x d y$.

The Fourier transforms of the elastic potentials have the following form:

$$
\begin{aligned}
\tilde{\Psi}_{i}=s^{-2} & a_{2 i-1}(\xi, \eta) \sinh \left(\gamma_{i}^{-1} s(h-z)\right)+ \\
& +s^{-2} a_{2 i}(\xi, \eta) \cosh \left(\gamma_{i}^{-1} s(h-z)\right), i=1,2,3 .
\end{aligned}
$$

The solution in the substrate can be expressed as:

$$
\begin{aligned}
& s^{2} \tilde{u}_{x}^{(0)}(\xi, \eta, z)=-i \xi \tilde{\theta}_{1}^{(0)}(\xi, \eta, z)-i \eta \tilde{\chi}^{(0)}(\xi, \eta, z), \\
& s^{2} \tilde{u}_{y}^{(0)}(\xi, \eta, z)=-i \eta \tilde{\theta}_{1}^{(0)}(\xi, \eta, z)+i \xi \tilde{\chi}^{(0)}(\xi, \eta, z), \\
& 2 \tilde{u}_{z}^{(0)}(\xi, \eta, z)=\left(d_{0} z a_{-1}(\xi, \eta)+2 a_{0}(\xi, \eta)\right) \exp (s z),
\end{aligned}
$$

where

$$
\begin{aligned}
& \begin{aligned}
2 \tilde{\theta}_{1}^{(0)}(\xi, \eta, z)=-\left[\left(2+d_{0}\right) a_{-1}(\xi, \eta)+d_{0} s z a_{-1}(\xi, \eta)+\right. \\
\left.+2 a_{0}(\xi, \eta) s\right] \exp (s z)
\end{aligned}, \\
& \tilde{\chi}^{(0)}(\xi, \eta, z)=a_{7}(\xi, \eta) \exp (s z) .
\end{aligned}
$$

Formulas (6) and (7) contain 9 unknown functions of the transform parameter. Satisfying boundary conditions we obtain two systems of algebraic equations to determine unknown functions. They contain 6 and 3 equations respectively:

$$
\begin{aligned}
& \left(A_{3}-A_{4} \kappa_{1} \gamma_{1}^{-2}\right) a_{2}(\xi, \eta)+\left(A_{3}-A_{4} \kappa_{2} \gamma_{2}^{-2}\right) a_{4}(\xi, \eta)=\tilde{p}(\xi, \eta) \\
& \left(\kappa_{1}+1\right) \gamma_{1}^{-1} a_{1}(\xi, \eta)+\left(\kappa_{2}+1\right) \gamma_{2}^{-1} a_{3}(\xi, \eta)=\frac{i \xi}{A_{5} s} \tilde{\tau}_{x}(\xi, \eta) \\
& \sum_{i=1}^{2} \kappa_{i} \gamma_{i}^{-1}\left(a_{2 i-1}(\xi, \eta) c_{i}+a_{2 i}(\xi, \eta) s_{i}\right)+a_{0} s=0 \\
& 2 \sum_{i=1}^{2}\left(a_{2 i-1}(\xi, \eta) s_{i}+a_{2 i}(\xi, \eta) c_{i}\right) \\
& -\left(2+d_{0}\right) a_{-1}(\xi, \eta)-2 a_{0}(\xi, \eta) s=0 \\
& \sum_{i=1}^{2}\left(A_{4} \kappa_{i} \gamma_{i}^{-2}-A_{3}\right)\left(a_{2 i-1}(\xi, \eta) s_{i}+a_{2 i}(\xi, \eta) c_{i}\right) \\
& -\mu_{0}\left(a_{-1}(\xi, \eta)+2 a_{0}(\xi, \eta) s\right)=0
\end{aligned}
$$

$$
\begin{aligned}
& A_{5} \sum_{i=1}^{2}\left(\kappa_{i}+1\right) \gamma_{i}^{-1}\left(a_{2 i-1}(\xi, \eta) c_{i}+a_{2 i}(\xi, \eta) s_{i}\right) \\
& +\mu_{0}\left(\left(1+d_{0}\right) a_{-1}(\xi, \eta)+2 a_{0}(\xi, \eta) s\right)=0, \\
& \gamma_{3}^{-1} a_{5}(\xi, \eta)=\frac{i \eta}{A_{5} s} \tilde{\tau}_{x}(\xi, \eta), \\
& a_{5}(\xi, \eta) s_{3}+a_{6}(\xi, \eta) c_{3}+b_{0}(\xi, \eta)=0,
\end{aligned}
$$

$A_{5} \gamma_{3}^{-1}\left(a_{5}(\xi, \eta) c_{3}+a_{6}(\xi, \eta) s_{3}\right)-\mu_{0} a_{7}(\xi, \eta)=0$.

where: $c_{i}=\cosh \left(\gamma_{i}^{-1} s h\right), s_{i}=\sinh \left(\gamma_{i}^{-1} s h\right), i=1,2,3$.

Solution of these two systems of equations can be normalized as follows:

$$
\begin{aligned}
a_{j}(\xi, \eta)=\frac{\tilde{p}(\xi, \eta)}{A_{5}} a_{j}^{(p)}(s)-\frac{i \xi \tilde{\tau}_{x}(\xi, \eta)}{s A_{5}} a_{j}^{(\tau)}(s), & \\
& i=-1,0, \ldots, 4,
\end{aligned}
$$

$a_{j}(\xi, \eta)=-\frac{i \eta \tilde{\tau}_{x}(\xi, \eta)}{s A_{5}} a_{j}^{(\tau)}(s), i=5,6,7$

Solving obtained linear equations and taking into account relationship between displacements and stresses and elastic potentials we obtain solution of the problem in Fourier transform domain.

$$
\begin{aligned}
& \tilde{\sigma}_{x x}^{(p)}=\left(S_{x y 1}^{(p)}(s, z)-\frac{\eta^{2}}{s^{2}} S_{x y 2}^{(p)}(s, z)\right) \tilde{p}(\xi, \eta), \\
& \tilde{\sigma}_{y y}^{(p)}=\left(S_{x y 1}^{(p)}(s, z)-\frac{\xi^{2}}{s^{2}} S_{x y 2}^{(p)}(s, z)\right) \tilde{p}(\xi, \eta),
\end{aligned}
$$

$$
\tilde{\sigma}_{z z}^{(p)}=-S_{z 1}^{(p)}(s, z) \tilde{p}(\xi, \eta)
$$

$\tilde{\sigma}_{x y}^{(p)}=\frac{\xi \eta}{s^{2}} S_{x y 2}^{(p)}(s, z) \tilde{p}(\xi, \eta)$,

$$
\tilde{\sigma}_{x z}^{(p)}=\frac{i \xi}{s} S_{z 2}^{(p)}(s, z) \tilde{p}(\xi, \eta),
$$

$\tilde{\sigma}_{y z}^{(p)}=\frac{i \eta}{s} S_{z 2}^{(p)}(s, z) \tilde{p}(\xi, \eta)$,

$\tilde{\sigma}_{x x}^{(\tau)}=$

$=-\frac{i \xi}{s}\left(S_{x y 1}^{(\tau)}(s, z)-\frac{\eta^{2}}{s^{2}}\left(S_{x y 2}^{(\tau)}(s, z)-2 S_{x y 3}^{(\tau)}(s, z)\right)\right) \tilde{\tau}_{x}(\xi, \eta)$,

$$
\begin{aligned}
\tilde{\sigma}_{y y}^{(\tau)}=- & \frac{i \xi}{s}\left[\left(S_{x y 1}^{(\tau)}(s, z)-S_{x y 2}^{(\tau)}(s, z)\right)+\right. \\
& \left.+\eta^{2} s^{-2}\left(S_{x y 2}^{(\tau)}(s, z)-2 S_{x y 3}^{(\tau)}(s, z)\right)\right] \tilde{\tau}_{x}(\xi, \eta),
\end{aligned}
$$

$\tilde{\sigma}_{z z}^{(\tau)}=\frac{i \xi}{s} S_{z 1}^{(\tau)}(s, z) \tilde{\tau}_{x}(\xi, \eta)$,

$\tilde{\sigma}_{x y}^{(\tau)}=$

$=-\frac{i \eta}{s}\left(S_{x y 3}^{(\tau)}(s, z)+\frac{\xi^{2}}{s^{2}}\left(S_{x y 2}^{(\tau)}(s, z)-2 S_{x y 3}^{(\tau)}(s, z)\right)\right) \tilde{\tau}_{x}(\xi, \eta)$, 


$$
\begin{aligned}
& \tilde{\sigma}_{x z}^{(\tau)}=\left(\frac{\xi^{2}}{s^{2}} S_{z 2}^{(\tau)}(s, z)+\frac{\eta^{2}}{s^{2}} S_{z 3}^{(\tau)}(s, z)\right) \tilde{\tau}_{x}(\xi, \eta), \\
& \tilde{\sigma}_{y z}^{(\tau)}=\frac{\xi \eta}{s^{2}}\left(S_{z 2}^{(\tau)}(s, z)-S_{z 3}^{(\tau)}(s, z)\right) \tilde{\tau}_{x}(\xi, \eta),
\end{aligned}
$$

where the form of introduced functions $S$ depends on the location of the investigated point. Moreover functions with subscripts $x y$ defined in the coating depend on the number of a sublayer in a fundamental layer and are given by:

$$
\begin{aligned}
& S_{x y 1}^{(j, k)}=\sum_{i=1}^{2} A_{5}^{-1}\left(M_{k} \kappa_{i} \gamma_{i}^{-2}-K_{k}\right)\left(a_{2 i-1}^{(j)} S_{i}+a_{2 i}^{(j)} C_{i}\right), j=p, \tau, k=1,2 \\
& S_{x y 2}^{(j, k)}=-2 A_{5}^{-1} \mu_{k} \sum_{i=1}^{2}\left(a_{2 i-1}^{(j)} S_{i}+a_{2 i}^{(j)} C_{i}\right), j=p, \tau, k=1,2, \\
& S_{x y 3}^{(\tau, k)}=-A_{5}^{-1} \mu_{k}\left(a_{5}^{(\tau)} S_{3}+a_{6}^{(\tau)} C_{3}\right), k=1,2, \\
& S_{z 1}^{(j)}=\sum_{i=1}^{2} A_{5}^{-1}\left(A_{3}-A_{4} \kappa_{i} \gamma_{i}^{-2}\right)\left(a_{2 i-1}^{(j)} S_{i}+a_{2 i}^{(j)} C_{i}\right), j=p, \tau, \\
& S_{z 2}^{(j)}=-\sum_{i=1}^{2}\left(\kappa_{i}+1\right) \gamma_{i}^{-1}\left(a_{2 i-1}^{(j)} C_{i}+a_{2 i}^{(j)} S_{i}\right), j=p, \tau, \\
& S_{z 3}^{(\tau)}=-\gamma_{3}^{-1}\left(a_{5}^{(\tau)} C_{3}+a_{6}^{(\tau)} S_{3}\right),
\end{aligned}
$$

where:

$$
S_{i}=\sinh \left(\gamma_{i}^{-1} s(h-z)\right), C_{i}=\cosh \left(\gamma_{i}^{-1} s(h-z)\right), i=1,2,3 .
$$

The corresponding functions in the substrate are as follows:

$$
\begin{aligned}
& \mu_{0}^{-1} A_{5} S_{x y 1}^{(j)}=-\left(\left(2 d_{0}+1\right) a_{-1}^{(j)}+d_{0} s z a_{-1}^{(j)}+2 a_{0}^{(j)} s\right) \exp (s z), \\
& \mu_{0}^{-1} A_{5} S_{x y 2}^{(j)}=-\left(\left(d_{0}+2\right) a_{-1}^{(j)}+d_{0} s z a_{-1}^{(j)}+2 a_{0}^{(j)} s\right) \exp (s z), \\
& \mu_{0}^{-1} A_{5} S_{z 1}^{(j)}=-\left(a_{-1}^{(j)}+d_{0} s z a_{-1}^{(j)}+2 a_{0}^{(j)} s\right) \exp (s z), \\
& \mu_{0}^{-1} A_{5} S_{z 2}^{(j)}=\left(\left(d_{0}+1\right) a_{-1}^{(j)}+d_{0} s z a_{-1}^{(j)}+2 a_{0}^{(j)} s\right) \exp (s z), j=p, \tau, \\
& \mu_{0}^{-1} A_{5} S_{x y 3}^{(\tau)}=-\mu_{0}^{-1} A_{5} S_{z 3}^{(\tau)}=a_{7}^{(\tau)} \exp (s z) .
\end{aligned}
$$

Taking the inverse Fourier integral transforms, we obtain relations (in terms of double integrals) between displacement vector components and stress tensor components in the coating and in the substrate and functions describing loading distributions.

\section{RESULTS AND DISCUSSIONS}

Calculations were made under assumptions:

- the tangential tractions are related to the normal tractions by the Amontons-Coulomb law of friction: $\tau_{x}=f p$ where $f$ is the friction coefficient

- the axisymmetrical pressure in the form $\left(\frac{p}{p_{0}}\right)^{2}=1-r^{2}$ $\left(r^{2}=x^{2}+y^{2}\right)$ is applied over a circular area of radius $a$.

- mechanical properties of the substrate and the coating as well as the thickness ratio of sublayers forming fundamental layer were taken from the literature (Voevodin et al., 2001). Details are given in Tab. 1.
Tab. 1. Mechanical and geometrical properties elements of the discussed non-uniform half space

\begin{tabular}{|c|c|c|c|c|}
\hline \multicolumn{2}{|c|}{ Material } & $E(\mathrm{GPa})$ & $v$ & $H_{1} / H_{2}$ \\
\hline \multirow{2}{*}{ substrate } & Steel & 220 & 0.25 & \multirow{4}{*}{$4.7 / 1.2$} \\
coating & $\mathrm{TiN}$ & 440 & 0.18 & \\
\cline { 2 - 4 } & $\mathrm{Ti}$ & 120 & 0.32 & \\
\hline
\end{tabular}

The integrals at internal points of the nonuniform half space $(z<h)$ are taken with the help of the Gaussian quadrature. On the surface $z=h$, we take into account the asymptotic behavior of the solution of the system of equations obtained as the parameter of the integral transformation tends to infinity. The integrals in which the integrands are replaced by their asymptotics are taken analytically. To find the remaining integrals, we apply the Gaussian quadrature.

Fig. 2 illustrates distributions of the first principal stress $\sigma_{1}$ in sublayers of the coating with greater Young modulus in the plane $y=0$. Figs. (2a) and (2b) show an interaction in case of normal traction. Contours of $\sigma_{1}$ distribution caused by tangential tractions are shown in Figs. (2c) and (2d). It can be seen that tensile stresses arise on the unloaded part of the surface of the half-space. The maximum value of $\sigma_{1}$ appears close to the point $x=-1, y=0, z=h$ and increases with increasing value of the friction coefficient. For specific thickness of the coating tensile stresses additionally appear in the vicinity of the coating-substrate interface.

Fig. 3 show distributions of the second invariant of deviatoric stress tensor $J_{2}\left(\sigma_{H M}=\sqrt{3} J_{2}\right)$ in sublayers with greater Young modulus. It can be distinguished two local maxima of $J_{2}$ : (1) at the point $x \approx-1, y=0, z=h$ (2) on the coating-substrate interface or in its vicinity.
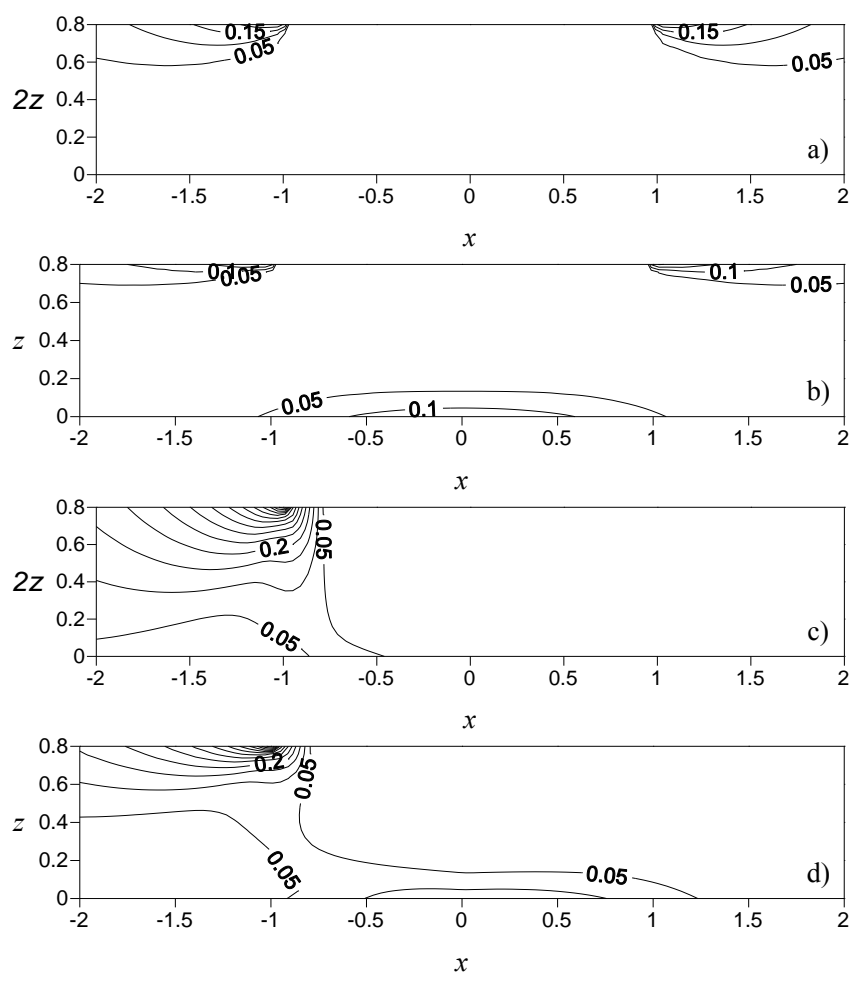

Fig. 2. Distribution of the first principal stress $\sigma_{1}$ in sublayers with greater Young modulus in regions in which $\sigma_{1}>0.05$ :

a) $h=0.4, f=0 ; b) h=0.8, f=0$; c) $h=0.4$, $f=0.25$; d) $h=0.8, f=0.25$. 

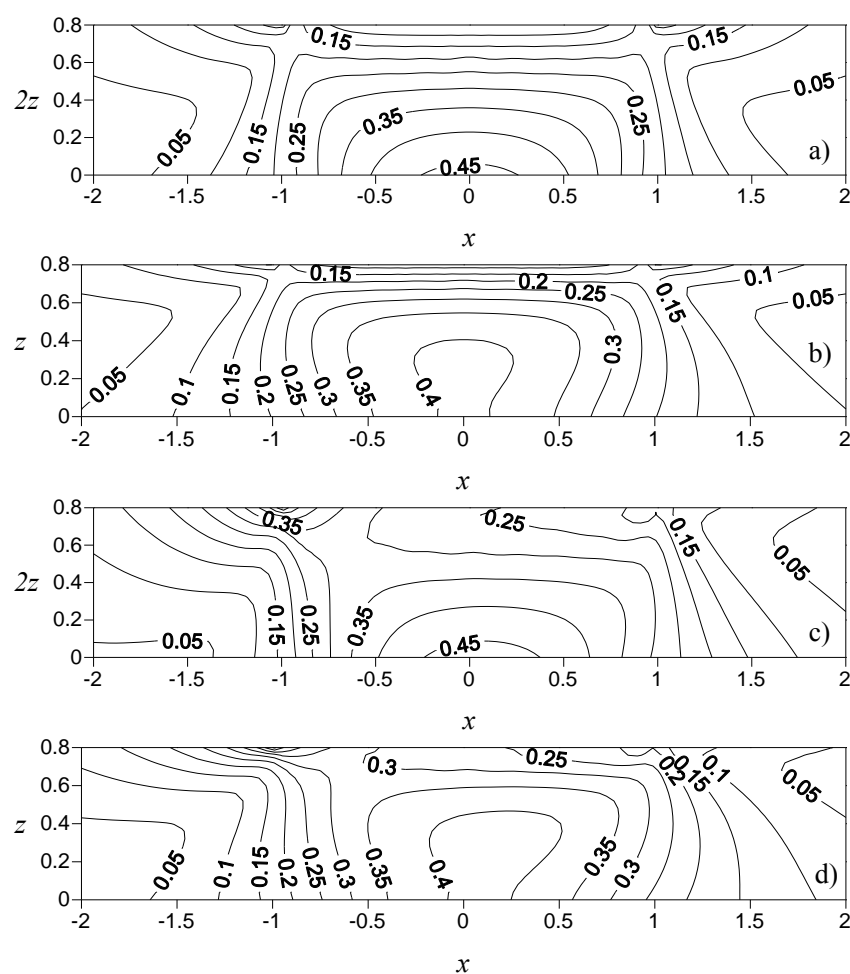

Fig. 3. Distributions of the second invariant of deviatoric stress tensor $J_{2}$ in sublayers with greater Young modulus: a) $h=0.4, f=0$;

b) $h=0.8, f=0$; c) $h=0.4, f=0.25$;

d) $h=0.8, f=0.25$

\section{CONCLUSIONS}

\section{Calculations show that:}

- Distribution of the first principal stress $\sigma_{1}$ in layers with greater Young modulus is similar to that in a homogeneous coating when $E_{\text {coating }}>E_{\text {substrate }}$ (Kulchytsky-Zhyhailo and Rogowski, 2007, 2010; Schwarzer, 2000). Values of tensile stresses (if exist) on the coating-substrate interface are lower than in a homogeneous coating with the same Young modulus.

- Distributions of the stress tensor components which experience a jump on the interfaces are different in layers with greater Young modulus from distributions in layers with smaller Young modulus.

\section{REFERENCES}

1. Bragallini G.M., Cavatorta M.P., Sainsot P. (2003), Coated Contacts: a Strain Approach, Tribology International, Vol. 36, 935941.

2. Elliot D.A. (1949), Three-Dimensional Stress Distributions in Aeolotropic Hexagonal Crystals, Cambridge Phil. Society, Vol. 44, 522-533.

3. Guler M.A., Erdogan F. (2007), The frictional Sliding Contact Problems of rigid parabolic and cylindrical stamps on Graded Coatings, Int. J. Mech. Sci., Vol. 49, 161-182.

4. Kaczyński A. (1994), Three-Dimensional Thermoelasic Problems of Interface Crack in Periodic Two-Layered Composites, Engineering Fracture Mechanics, Vol. 48, 783-800.

5. Kołodziejczyk W., Kulchytsky-Zhyhailo R. (2013), Stress Distribution in Two-Layered Half-Space with Periodical Structure Caused by Hertz Pressure, J. Theor. Appl. Mech.,Vol.51, 741-750.

6. Kulchytsky-Zhyhailo R. (2011), Two-Layered Periodic Coated Elastic Half-Space under Hertz's Pressure, Materials Science, Vol. 47, No. 4, 527-534.

7. Kulchytsky-Zhyhailo R., Bajkowski A. (2011), Elastic Half Space with Inhomogeneous Coating under the Action of Tangential Forces, Materials Science, Vol. 46, 735-746.

8. Kulchytsky-Zhyhailo R., Kolodziejchyk V. (2005), Stress Field Caused by Hertz's Pressure in non-uniform half-plane with Periodic Structure, J. Friction and Wear, Vol. 26, No. 4, 358-366.

9. Kulchytsky-Zhyhailo R., Rogowski G. (2007), Stresses of Hard Coating under Sliding Contact, J. Theor. Appl. Mech., Vol. 45, 753-771.

10. Kulchytsky-Zhyhailo R., Rogowski G. (2010), Stresses in hard coating due to a rigid spherical indenter on a Layered Elastic HalfSpace, Tribology Int., Vol. 43, No. 9, 1592-1601.

11. Liu T.J., Wang Y.S., Zhang C. (2008), Axisymmetric Frictionless Contact of Functionally Graded Materials, Archive of Appl. Mech., Vol. 78, 267-282.

12. Matysiak S.J., Woźniak Cz. (1987), Micromorphic Effects in Modelling of Periodic Multilayered Elastic Composites, Int. J. Engng. Sci., Vol. 25, 549-559.

13. Schwarzer N. (2000), Coating Design Due to Analytical Modelling of Mechanical Contact Problems of Multilayer Systems, Surf. Coat. Technol., Vol. 133-134, 397-402.

14. Voevodin A.A., larve E.V., Ragland W., Zabinski J.S., Donaldson S. (2001), Stress analyses and In-Situ Fracture Observation of Wear Protective Multilayer Coatings in Contact Loading, Surf. Coat. Technol., Vol. 148, 38-45.

15. Woźniak Cz. (1987), A nonstandard method of Modelling Thermoelastic Composites, Int. J. Engng. Sci., Vol. 25, 483-499.

The investigations described in this paper are a part of the research projects S/WM/1/2013 and S/WM/1/2012 realized at Bialystok University of Technology. 